\title{
Métricas de paisagem na avaliação da efetividade de proteção do Parque Estadual da Costa do Sol, uma unidade de conservação fragmentada no Estado do Rio de Janeiro, Brasil
}

\section{Landscape metrics in the evaluation of the protective effectiveness of the State Park of Costa do Sol, a fragmented conservation unit in the state of Rio de Janeiro, Brazil}

\author{
Georg de Oliveira Müller ${ }^{1}$, Luciana Mara Temponi de Oliveira ${ }^{2}$
}

1 Programa de Pós Graduação em Ciências Ambientais e Conservação - PPGCiAC, Universidade Federal do Rio de Janeiro - UFRJ, Instituto de Biodiversidade e Sustentabilidade (NUPEM), Avenida São José Barreto, 764, CEP 27971-550, Macaé, RJ, Brasil

2 Instituto Brasileiro de Geografia e Estatística - IBGE, Diretoria de Geociências, Avenida República do Chile, 500 - $7^{\circ}$ andar, CEP 20031-170, Rio de Janeiro, RJ, Brasil

Corresponding author: Georg de Oliveira Müller (georg.muller@hotmail.com)

Academic editor:A.M. Leal-Zanchet | Received 26 July 2019 | Accepted 18 November 2019 | Published 6 Febaruary 2020 Citation: Müller GO, Oliveira LMT (2020) Métricas de paisagem na avaliação da efetividade de proteção do Parque Estadual da Costa do Sol, uma unidade de conservação fragmentada no Estado do Rio de Janeiro, Brasil. Neotropical Biology and Conservation 15(1): 1-18. https://doi.org/10.3897/neotropical.15.e49490

\section{Resumo}

A região de Cabo Frio e seu entorno, no litoral do Estado do Rio de Janeiro, Brasil, apresenta alta riqueza de espécies vegetais e um grande número de endemismos, o que a torna uma área prioritária para a conservação, sendo conhecida como o Centro de Diversidade Vegetal de Cabo Frio (CDVCF). O Parque Estadual da Costa do Sol (PECSol) é a maior Unidade de Conservação de Proteção Integral da região e foi concebido num modelo descontínuo, com 43 áreas distintas pertencentes a quatro grandes núcleos: Massambaba, Atalaia-Dama Branca, Pau-Brasil e Sapiatiba. Dada a natureza fragmentada do PECSol, este estudo teve como objetivo avaliar, através de métricas de paisagem, a efetividade do PECSol como elemento protetor da biodiversidade do CDVCF, considerando seus 43 
fragmentos como eventuais remanescentes únicos de áreas naturais na região. Os resultados apontaram que os fragmentos do PECSol sofrem forte pressão de atividades antrópicas em sua vizinhança imediata, e estão, de maneira geral, altamente suscetíveis a efeitos de borda. A conectividade entre os fragmentos é em geral baixa, tendo os fragmentos do núcleo Massambaba apresentado os melhores indicadores de disponibilidade de habitat, com valores entre 0,18 e 0,57 num índice que varia de 0 a 1. Nenhum outro fragmento apresentou valor acima de 0,10 . Outras quatro grandes áreas naturais da região - a Ilha de Cabo Frio, a porção vegetada da Base Aérea Naval de São Pedro da Aldeia, a área de restinga na Estação Radiogoniométrica da Marinha em Campos Novos e as Dunas do Peró - apresentam grande importância para a disponibilidade de habitat no CDVCF. A inclusão destas áreas no PECSol ou em outra Unidade de Conservação de Proteção Integral é recomendada.

\section{Abstract}

The Cabo Frio region and its surroundings, in the state of Rio de Janeiro's coast, Brazil, is an area with high plant species richness and high endemism, which makes it a priority area for conservation, known as the Centre of Plant Diversity of Cabo Frio (CPDCF). The State Park of Costa do Sol (SPCSol) is the largest conservation unit with integral protection in the region and was developed in a discontinuous model, being formed by 43 distinct areas grouped in four large nuclei: Massambaba, Atalaia-Dama Branca, Pau-Brasil and Sapiatiba. Given the fragmented aspect of the SPCSol, the goal of the present study was to evaluate, through landscape metrics, the effectiveness of the SPCSol as a protective tool for the CPDCF's biodiversity, accounting its 43 fragments as eventual unique remnants of natural areas in the region. Results showed that the SPCSol's fragments are under strong pressure from anthropic activities in its direct vicinity, being in general highly susceptive to edge effects. The connectivity between fragments is low, with the Massambaba nucleus presenting the best values of habitat availability, varying between 0.18 and 0.57 in an index that ranges from 0 to 1 . None of the other fragments presented values above 0.10 . Another four large natural areas in the region - the Cabo Frio Island, the vegetated portion of São Pedro da Aldeia’s Naval Air Base, the "restinga" area in the Navy's Radio Direction Finding Station in Campos Novos and the Peró Dunes - showed high importance for habitat availability in the CPDCF. The inclusion of these areas in the SPCSol or in another conservation unit with integral protection is recommended.

\section{Palavras-chave}

Centro de Diversidade Vegetal de Cabo Frio, ecologia de paisagens, fragmentação de habitats

\section{Keywords}

Centre of Plant Diversity of Cabo Frio, habitat fragmentation, landscape ecology

\section{Introdução}

A destruição de habitats naturais, em geral para o desenvolvimento de atividades humanas, é a maior causa de perda de biodiversidade nos dias atuais (Pimm e Raven 2000). A teoria de biogeografia de ilhas de MacArthur e Wilson (1967), aplicável a fragmentos de áreas naturais por funcionarem como ilhas de habitat em meio a ambientes modificados, relaciona a riqueza de espécies de uma determinada ilha a seu tamanho e ao seu grau de isolamento, fatores que influenciam no equilíbrio entre suas taxas de extinção e imigração, sendo aquelas de maior tamanho e as mais próximas de outras áreas naturais as que comportam uma maior riqueza. 
Além dos efeitos de área e de distância consolidados pela teoria da biogeografia de ilhas, outro fator que influencia na riqueza de espécies de fragmentos de habitat são os efeitos de borda. Nas porções limítrofes entre fragmentos de vegetação e as áreas modificadas no seu entorno há alterações graduais em fatores físicos como incidência de radiação solar, umidade, temperatura e exposição aos ventos, o que interfere na sobrevivência de espécies menos generalistas nestas áreas de borda, reduzindo sua riqueza (Lovejoy et al. 1986).

Outro aspecto determinante na riqueza de um fragmento de habitat é sua conectividade com outros fragmentos. Taylor et al. (1993) definem conectividade como "o grau no qual uma paisagem facilita ou restringe o movimento dos organismos entre fragmentos de habitat". Pascual-Hortal e Saura (2006) criticam essa definição clássica de conectividade considerando-a muito reducionista, levando à interpretação equivocada de que 100 fragmentos interligados de 1 hectare possuem conectividade muito maior que um grande fragmento com 100 hectares contínuos de habitat. Estes autores compreendem os fragmentos em si como uma área onde a conectividade ocorre, e propõem que esta seja entendida como "disponibilidade de habitat", incorporando a ela a dimensão de área (Pascual-Hortal e Saura 2006; Saura e Rubio 2010).

A crescente fragmentação dos habitats naturais e a necessidade de esforços mais efetivos de conservação da biodiversidade levaram ao surgimento da Ecologia de Paisagens. Essa ciência interdisciplinar busca compreender a influência dos padrões espaciais nos processos ecológicos e enxerga o conceito de paisagem em diferentes escalas espaciais e temporais (Metzger 2001). Com a ajuda de imagens de satélite e sensoriamento remoto, além da popularização dos computadores e de Sistemas de Informação Geográfica, cada vez mais estudos de Ecologia de Paisagens fazem uso de fórmulas e equações matemáticas para identificar e compreender os padrões espaciais de uma determinada paisagem (métricas de paisagem), cada qual com potencialidades e limites de aplicabilidade e interpretação diversos (Hargis et al. 1998; Li e Wu 2004), dando suporte aos esforços de conservação de espécies e ao planejamento de áreas protegidas.

No Estado do Rio de Janeiro, na porção da costa conhecida como Região dos Lagos, encontra-se o Centro de Diversidade Vegetal de Cabo Frio (CDVCF). Descrito por Araujo (1997), o CDVCF é uma área que apresenta alta heterogeneidade climática, geomorfológica e de solos, o que se reflete numa grande variedade de espécies vegetais, com riqueza estimada de 1.500 a 2.200 espécies, além de um grande número de espécies endêmicas. A região encontra-se consideravelmente antropizada (58,1\% de sua área) (Bohrer et al. 2009), com extensas áreas de plantio e pasto ao norte, inúmeras salinas e grandes manchas urbanas no entorno da Lagoa de Araruama. As áreas naturais remanescentes estão fragmentadas e são ameaçadas pela introdução de espécies exóticas, como Casuarina equisetifolia Linnaeus, 1759 (Zimmermann 2016), e pela supressão da vegetação natural para expansão urbana e implementação de salinas, plantios, pastagens e empreendimentos turísticos de grande porte (Araujo 1997; Bohrer et al. 2009; Lerner e Jeronymo 2017). 
A região do CDVCF possui diversas Unidades de Conservação, sendo sua maioria Unidades de Uso Sustentável. A principal Unidade de Proteção Integral da região é o Parque Estadual da Costa do Sol (PECSol). Criado em 2011 com o objetivo de proteger os ecossistemas remanescentes da região e os elementos característicos de sua paisagem, o PECSol segue um modelo de parque não-contínuo, sendo formado por 43 fragmentos distintos, perfazendo uma área total aproximada de $98,4 \mathrm{~km}^{2}$. Este modelo de parque fragmentado, porém, não é explicitamente previsto pelo Sistema Nacional de Unidades de Conservação (SNUC) (Brasil 2000), e a opção do Poder Público em adotá-lo gerou críticas pela comunidade científica (Corrêa e Fontenelle 2012).

Considerando a natureza fragmentada do Parque Estadual da Costa do Sol e a biodiversidade única do Centro de Diversidade Vegetal de Cabo Frio, este estudo teve como objetivo realizar uma avaliação quantitativa, por meio de métricas de paisagem, e qualitativa da efetividade do PECSol em preservar a biodiversidade do CDVCF a longo prazo num cenário hipotético onde as áreas por ele protegidas sejam as últimas áreas naturais remanescentes na região. A pesquisa tem ainda como objetivos específicos: identificar o grau de pressão que sofrem as áreas do PECSol pelas atividades antrópicas em sua vizinhança; avaliar a suscetibilidade das áreas do PECSol a efeitos de borda; verificar o grau de isolamento das áreas do PECSol entre si e apontar aquelas que melhor contribuem para a conectividade dos remanescentes vegetacionais do CDVCF; e avaliar a importância de outras áreas naturais da região não protegidas pelo PECSol.

\section{Material e métodos}

\section{Caracterização da área de estudo}

A área de estudo é o Centro de Diversidade Vegetal de Cabo Frio (CDVCF), conforme descrito por Araujo (1997) e mapeado por Bohrer et al. (2009). Está situado no Estado do Rio de Janeiro, Brasil, entre as coordenadas $22^{\circ} 30^{\prime} \mathrm{S}$ a $23^{\circ} 00^{\prime} \mathrm{S}$ e $41^{\circ} 52^{\prime} \mathrm{O}$ a $42^{\circ} 42^{\prime} \mathrm{O}$. Compreende, em partes ou em sua totalidade, os municípios de Maricá, Saquarema, Araruama, Iguaba Grande, São Pedro da Aldeia, Arraial do Cabo, Cabo Frio e Armação dos Búzios. Com área de aproximadamente $1.500 \mathrm{~km}^{2}$, é delimitado a oeste pela Serra do Mato Grosso, a sul e a leste pelo Oceano Atlântico e a norte pelos rios Una e São João.

O CDVCF possui menos de $10 \%$ de sua área com altitudes acima de $100 \mathrm{~m}$, atingindo máxima de cerca de 500m (Araujo 1997). A paisagem é dominada pelas planícies litorâneas, com presença de alguns maciços costeiros. A região foi modelada por uma sucessão de subidas e recuos no nível do mar durante o Quaternário, que deu origem aos depósitos sedimentares que compõem as planícies litorâneas, bem como a um complexo lagunar hipersalino com lagunas maiores, como a de Araruama, mais internas e outras menores, mais externas, separadas entre si e do oceano por duas faixas de cordões arenosos, com ocorrência de grandes campos de dunas, como as de Tucuns, Peró, Dama Branca e Massambaba (Coe et al. 2007). 
Seu clima é semi-árido quente, em contraste com o clima tropical úmido predominante no resto do Estado. Sua precipitação anual média é de cerca de $800 \mathrm{~mm}$, valor bem abaixo das demais áreas costeiras do Estado, que possuem média de $1.200 \mathrm{~mm}$, podendo chegar a mais de $2.000 \mathrm{~mm}$ em Angra dos Reis. A evaporação média é de 1.200 a $1.400 \mathrm{~mm} /$ ano (Barbiére 1975). A temperatura varia de $11,2^{\circ} \mathrm{C}$ a $36,4^{\circ} \mathrm{C}$, com média anual entre $20,9^{\circ} \mathrm{C}$ e $25,1^{\circ} \mathrm{C}$. A umidade relativa varia de $81 \%$ a $85 \%$ (Barbiére 1997). Na região predominam na maior parte do ano os ventos do setor NE, com maior intensidade no verão. No inverno, ganham força os ventos dos setores S e SO, geralmente associados à entrada de frentes frias (Barbiére 1975).

A vegetação se apresenta de forma heterogênea ao longo da paisagem. Nas áreas baixas mais próximas ao mar há formações de restingas, com estratos herbáceos, arbustivos e arbóreos sobre solos arenosos. Nas áreas mais interioranas encontra-se vegetação ripária junto aos leitos dos rios e em depressões alagáveis, e formações de florestas estacionais, como nas Serras de Sapiatiba e das Emerenças. A floresta ombrófila está presente nas áreas que demarcam os limites externos do CDVCF, onde o clima faz a transição para o tropical úmido, como na Serra do Mato Grosso. Há alguns pequenos manguezais, estando o mais expressivo próximo à desembocadura do rio São João. Sobre os maciços costeiros desde a península de Búzios até a Ilha de Cabo Frio encontra-se uma vegetação com fitofisionomias de savana estépica, com muitas plantas xerofíticas, adaptadas à escassez hídrica, com destaque às famílias Cactaceae e Bromeliaceae (Bohrer et al. 2009). Ab'Saber (1977) considera a região de Cabo Frio como um reduto remanescente de um clima seco que recobria grande parte da América do Sul após a última glaciação do Quaternário, onde predominariam as formações de caatingas.

No CDVCF está situado o Parque Estadual da Costa do Sol (PECSol), maior Unidade de Conservação de Proteção Integral da região. Com área total aproximada de $98,4 \mathrm{~km}^{2}$, o PECSol é formado por 43 fragmentos distintos (Figura 1), agrupados em quatro grandes núcleos: Massambaba, Atalaia-Dama Branca, Pau-Brasil e Sapiatiba, espalhados pelos municípios de Saquarema, Araruama, São Pedro da Aldeia, Arraial do Cabo, Cabo Frio e Armação dos Búzios.

\section{Metodologia}

Por meio do programa QGIS, versão 2.18, criou-se um projeto onde foram adicionadas as camadas vetoriais com as áreas do Parque Estadual da Costa do Sol e de sua Zona de Amortecimento, além do mapeamento de cobertura e uso da terra no CDVCF de Bohrer et al. (2009). Utilizou-se por padrão a projeção cônica equivalente de Albers, a fim de se obterem valores mais precisos de área para os cálculos das métricas. Os parâmetros dessa projeção adotados para a área de estudo foram obtidos seguindo Deetz e Adams (1921).

Para analisar o grau de pressão sofrido pelos fragmentos do PECSol pelas atividades antrópicas em sua vizinhança optou-se por identificar as classes de cobertura e uso da terra que ocorrem em sua Zona de Amortecimento (Figura 2). O SNUC define Zona de Amortecimento (ZA), como "o entorno de uma unidade de con- 

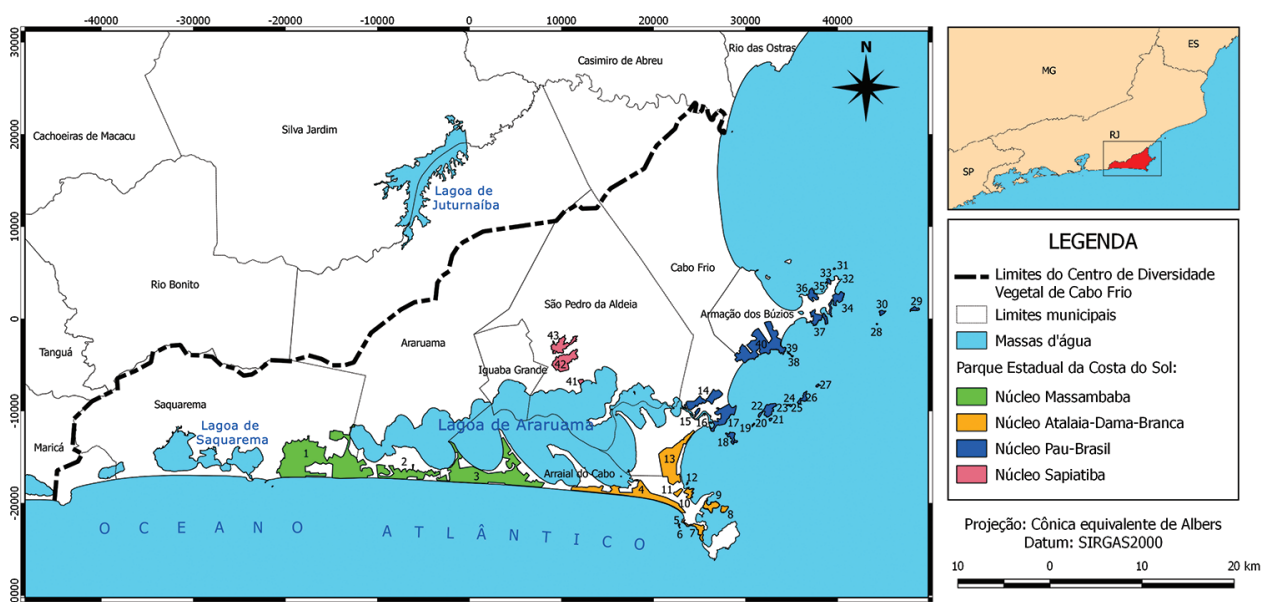

Figura 1. Localização das áreas do Parque Estadual da Costa do Sol no Centro de Diversidade Vegetal de Cabo Frio, Rio de Janeiro, Brasil.

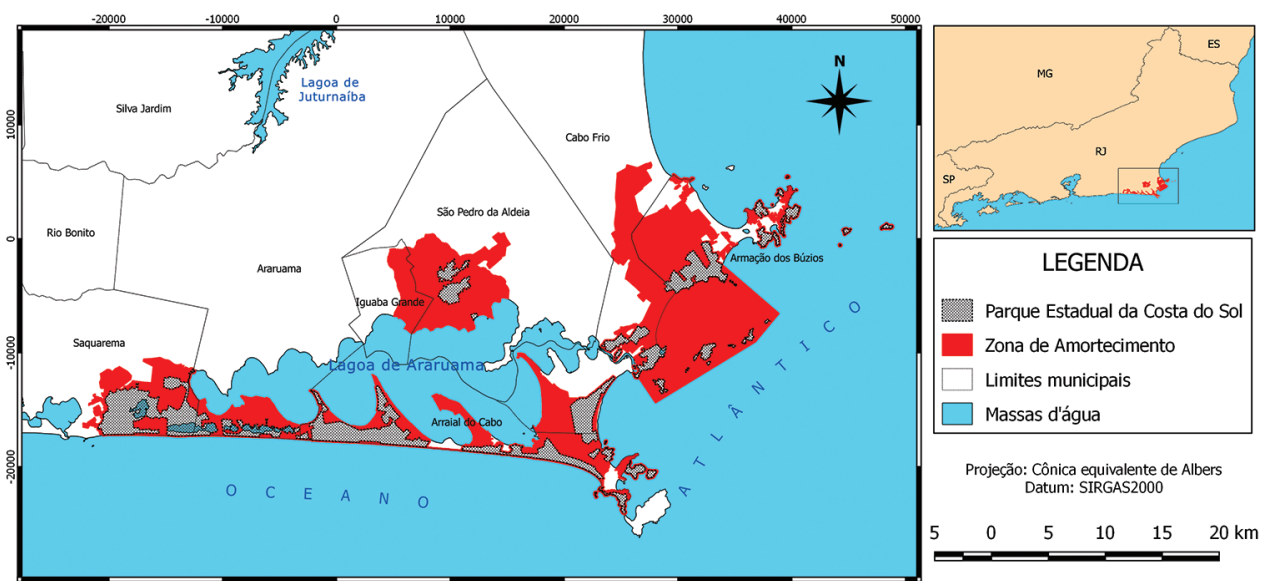

Figura 2. Zona de Amortecimento do Parque Estadual da Costa do Sol, Rio de Janeiro, Brasil.

servação, onde as atividades humanas estão sujeitas a normas e restrições específicas, com o propósito de minimizar os impactos negativos sobre a unidade" (Brasil 2000). O decreto de criação do PECSol define que sua ZA deverá ser definida em seu plano de manejo (Rio de Janeiro 2011), que só foi aprovado pela Resolução Inea $\mathrm{n}^{\circ} 181$ de 14 de junho de 2019, mais de oito anos após sua criação.

Para calcular a proporção de cada classe de uso dentro da ZA, sobrepôs-se o vetor da ZA ao mapeamento de cobertura e uso da terra de Bohrer et al. (2009), obtendo-se uma camada vetorial de interseção entre as duas. Utilizou-se a calculadora de campo na tabela de atributos da nova camada obtida para calcular a área de cada polígono distinto de cobertura e uso da terra na ZA do PECSol, permitindo assim obter a razão das áreas de cada classe em relação à área total da ZA. 
Para avaliar o grau de suscetibilidade a efeitos de borda dos fragmentos do PECSol, calculou-se a área e o perímetro de cada um deles. A partir dos valores obtidos, calculou-se o Índice de Circularidade (IC) de cada fragmento. O IC é comumente usado em estudos de bacias hidrográficas e de fragmentação de habitats (Li et al. 2013), e baseia-se na desigualdade isoperimétrica de Osserman (1978), com a seguinte fórmula:

$$
I C=\frac{4 \pi A}{P^{2}}
$$

$\mathrm{Na}$ qual $A$ é a área do fragmento e $P$ seu perímetro. Esse índice retorna valor 1 para formas perfeitamente circulares e valores entre 0 e 1 para polígonos não circulares, aproximando-se de zero conforme tornam-se mais alongados. Quanto mais irregular for a forma de um fragmento, maior será sua extensão de bordas e menor será a sua área central livre dos efeitos de borda (Laurance e Yensen 1991). Baseando-se no IC, dividiram-se os fragmentos do PECSol em três classes de suscetibilidade a efeitos de borda: maior, intermediária e menor, obtidas pelo método de quebras naturais de Jenks (1967), calculado no QGIS.

Para avaliar a conectividade entre os fragmentos, utilizou-se o Índice Integral de Conectividade (IIC) (Pascual-Hortal e Saura 2006), conforme a fórmula:

$$
I I C=\frac{\sum_{i=1}^{n} \sum_{j=1}^{n} \frac{a_{i} \cdot a_{j}}{1+n l_{i j}}}{A_{L}^{2}}
$$

Em que $a$ é a área de cada fragmento, $n l_{i j}$ é o número de ligações (links) no menor caminho (distância topológica) entre os fragmentos $i$ e $j$ (com valor 0 quando não interligados) e $A_{L}$ é a área da paisagem (todo o mosaico, incluindo habitat e nãohabitat). Esse índice retorna valores entre 0 e 1 , sendo 1 correspondente ao caso hipotético em que a paisagem inteira é preenchida por habitat (homogênea), e tendendo a 0 quanto menor for a disponibilidade de habitat (por área e por conectividade).

Para o cálculo do IIC utilizou-se o programa CONEFOR SENSINODE, versão 2.6. Os parâmetros utilizados foram a área dos fragmentos, os valores da menor distância euclidiana entre cada fragmento do PECSol e os demais, a área total da paisagem e a distância máxima de dispersão de organismos, cujo valor define os pares de fragmentos com uma distância entre si igual ou menor a este valor como funcionalmente conectados. A área dos fragmentos foi obtida anteriormente no QGIS e os valores da menor distância euclidiana entre os fragmentos foram obtidos por meio do plugin do CONEFOR para o QGIS. Para a área total da paisagem, foi considerada a área aproximada do CDVCF de $1.500 \mathrm{~km}^{2}$. Quanto ao valor máximo de dispersão de organismos, como este estudo buscou avaliar a conectividade do PECSol para a biodiversidade do CDVCF como um todo, e não para uma determinada espécie, adotou-se uma metodologia similar à de García-Feced et al. (2011), trabalhando-se com cinco valores referentes a espécies hipotéticas com capacidades 
de dispersão distintas: $50 \mathrm{~m}, 100 \mathrm{~m}, 500 \mathrm{~m}, 1.000 \mathrm{~m}$ e $2.000 \mathrm{~m}$, calculando-se assim cinco vezes o IIC e obtendo-se a média destes valores.

O programa CONEFOR fornece também a variação no IIC (dIIC) promovida pela retirada de um fragmento do cálculo do IIC, através da fórmula:

$$
d I I C=100 \times \frac{I I C-I I C^{\prime}}{I I C}
$$

Sendo IIC' um novo valor de IIC obtido após a remoção de um determinado fragmento. Este índice mostra, em porcentagem, o quanto a perda de um fragmento reduziria a conectividade e disponibilidade de habitat da paisagem, e pode ser interpretado como a importância individual de cada fragmento para a conectividade (Pascual-Hortal e Saura 2006). Também foi tirada a média dos cinco dIICs obtidos com os valores de dispersão distintos para cada um dos 43 fragmentos do PECSol. Estes, por sua vez, foram divididos em cinco classes de importância: muito baixa, baixa, média, alta e muito alta, classificação também realizada pelo método de quebras naturais de Jenks (1967) no QGIS.

Realizou-se uma segunda análise de conectividade onde foram incluídas outras quatro áreas do CDVCF que não são protegidas por Unidades de Conservação de Proteção Integral, mas que possuem grandes porções de vegetação remanescente, sendo elas: a Ilha de Cabo Frio, em Arraial do Cabo; a porção vegetada da Base Aérea Naval de São Pedro da Aldeia (BAeNSPA); a área de restinga arbórea da Estação Radiogoniométrica da Marinha em Campos Novos (ERMCN), no distrito de Tamoios em Cabo Frio; e as Dunas do Peró, em Cabo Frio. Para efeito de identificação, estas áreas receberam os números 44, 45, 46 e 47, respectivamente, seguindo a numeração dos fragmentos do PECSol. Os polígonos destas áreas foram manualmente vetorizados sobre imagens de satélite DigitalGlobe, visualizadas com o plugin "OpenLayers" do QGIS.

O segundo cálculo do IIC seguiu a mesma metodologia do cálculo anterior, sendo que as quatro áreas adicionais foram incluídas através da opção "there are nodes to add" do CONEFOR. Assim, o cálculo de dIIC desses fragmentos tem a sua lógica invertida, medindo o acréscimo na conectividade da paisagem que a adição desses fragmentos promoveria, sendo assim possível mensurar o benefício que sua proteção traria para além daqueles protegidos pelo PECSol.

Um resumo de toda a metodologia utilizada neste estudo é apresentado na Figura 3.

\section{Resultados e discussão}

\section{Análise de vizinhança na Zona de Amortecimento}

As classes de uso da terra mais expressivas identificadas na ZA do PECSol foram: corpos d’água (26,06\%), atividades agropecuárias $(24,74 \%)$ e áreas urbanas $(16,34 \%)$. A grande proporção de corpos d'água se dá pela proximidade dos fragmentos do 


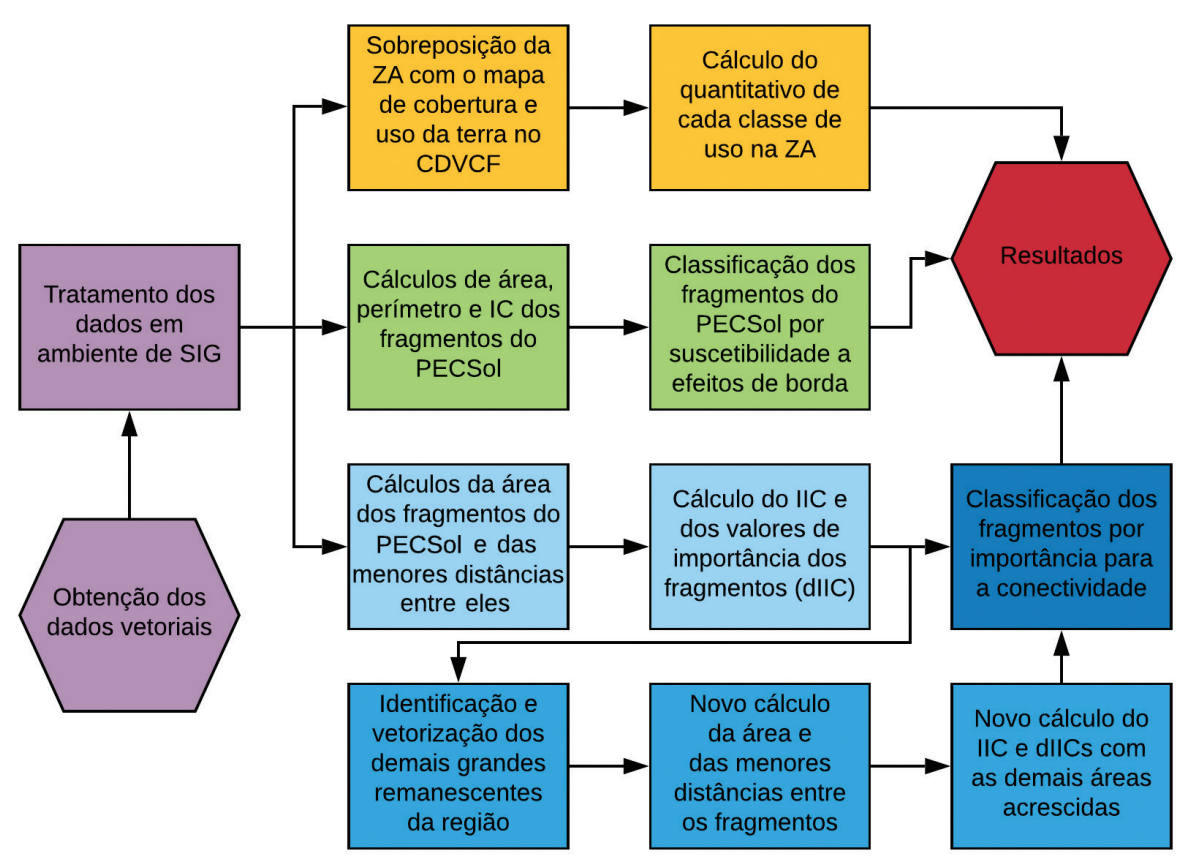

Figura 3. Resumo da metodologia adotada para avaliação da efetividade de proteção do Parque Estadual da Costa do Sol (PECSol), Centro de Diversidade Vegetal de Cabo Frio (CDVCF), Rio de Janeiro, Brasil. IC: Índice de Circularidade; IIC: Índice Integral de Conectividade; SIG: Sistema de Informação Geográfica; ZA: Zona de Amortecimento.

PECSol tanto do mar quanto do sistema lagunar da região. A Área de Proteção Ambiental Estadual do Pau-Brasil, outra UC da região que foi definida como uma das áreas que compõem a ZA do PECSol (Rio de Janeiro 2011), responde pela maior parte desta classe, devido a seus limites englobarem uma grande área oceânica em frente ao Peró (ver Figura 2). As classes correspondentes a atividades antrópicas respondem juntas pela maior porção de uso da terra na ZA (Figura 4A). Estas ficam em maior evidência quando retiram-se do cálculo os corpos d'água, somando $66,68 \%$ da área da ZA (Figura 4B).

Esses dados revelam que há poucos remanescentes de vegetação no entorno do PECSol e muitas áreas antropizadas imediatamente adjacentes a ele, em especial áreas urbanas e pastagens, com destaque também para a grande presença de salinas, setor mais forte da economia da região após o turismo. Vários dos fragmentos do PECSol estão dentro do perímetro urbano de algumas das principais cidades da região, como Cabo Frio e Búzios. Destaca-se que esta análise foi gerada sobre o mapeamento de cobertura uso da terra de Bohrer et al. (2009) que, apesar de ser o mapeamento mais detalhado em escala e classes de uso para a região do CDVCF, foi realizado há mais de 10 anos e pode não representar fielmente a situação atual da região. 
A

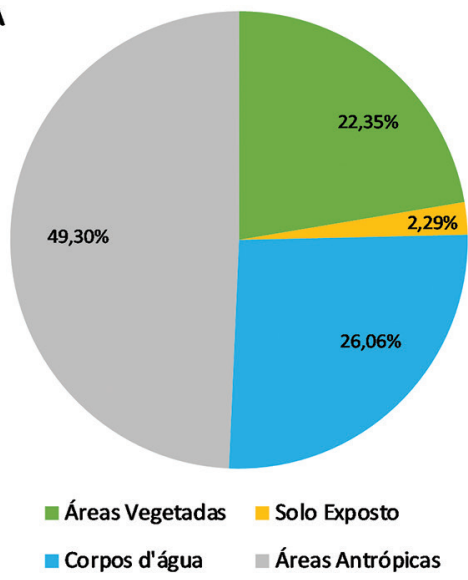

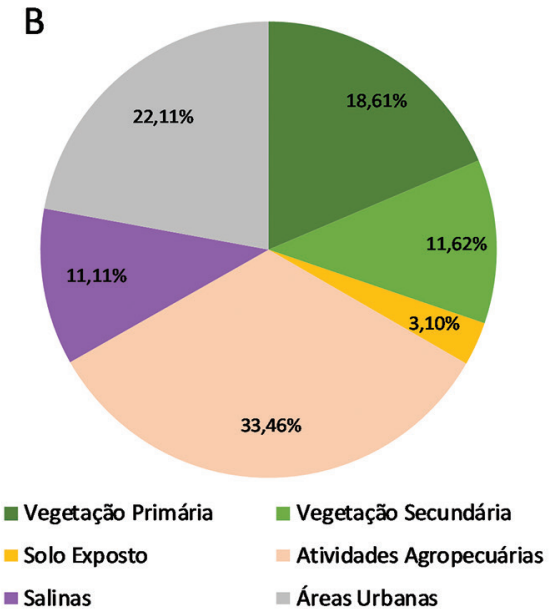

Figura 4. Proporção das classes de uso da terra na Zona de Amortecimento do Parque Estadual da Costa do Sol, Rio de Janeiro, Brasil. A divisão em quatro grandes classes: Áreas Vegetadas, que englobam todas as áreas com cobertura vegetal; Corpos d’Água; Solo Exposto, que incluiu dunas e praias; e Áreas Antrópicas, formadas por Atividades Agropecuárias, Áreas Urbanas e Salinas. B proporções de uso desconsiderando os Corpos d’Água, com as Áreas Vegetadas dividas em Vegetação Primária e Vegetação Secundária, e Áreas Antrópicas divididas em Atividades Agropecuárias, Áreas Urbanas e Salinas.

A disposição dos remanescentes vegetacionais em meio a um mosaico de usos da terra diversificados também justifica a escolha do Poder Público pelo modelo de parque descontínuo, visto que já não havia mais nenhuma grande área natural remanescente contínua em escala compatível à do Centro de Diversidade Vegetal de Cabo Frio. Entretanto, áreas protegidas cercadas pela presença de populações humanas estão sujeitas a diversos impactos (Wittemyer et al. 2008). A análise aqui apresentada da Zona de Amortecimento do PECSol evidencia a grande pressão que as atividades antrópicas exercem sobre seus fragmentos. A proximidade a essas atividades deixa-os mais suscetíveis a eventos como invasão de espécies exóticas, incêndios, contaminação do solo e do lençol freático, além da retirada da vegetação para a abertura de loteamentos ilegais.

\section{Suscetibilidade a efeitos de borda}

O IC médio dos 43 fragmentos do PECSol é de 0,45 , dentro da faixa intermediária de suscetibilidade a efeitos de borda, conforme observado na Figura 5. Destaca-se, entretanto, que dos 43 fragmentos, 23 correspondem a ilhas (ver Tabela 1). Essas ilhas detêm os maiores valores de IC dentre os fragmentos do PECSol, tendo apenas duas delas grau maior de suscetibilidade a efeitos de borda (fragmentos 18 e 26). De forma inversa, apenas dois dos fragmentos localizados em porção terrestre apresentam grau menor de suscetibilidade aos efeitos de borda (fragmentos 11 e 41).

Considerando apenas as 20 áreas do PECSol localizadas em porções terrestres, o IC médio cai para 0,277, valor limítrofe entre as classes de maior e intermediária suscetibilidade a efeitos de borda. Essa diferença nos ICs entre fragmentos de ilhas 
Tabela 1. Resumo das métricas (área, perímetro, Índice de Circularidade - IC - e valores de importância do Índice Integral de Conectividade para os cinco limiares de dispersão calculados - dIIC50 a dIIC2000 - e a média desses valores) de cada fragmento do Parque Estadual da Costa do Sol (1-43), e das outras quatro áreas naturais (fragmentos 44-47) analisadas no Centro de Diversidade Vegetal de Cabo Frio, Rio de Janeiro, Brasil. *: ilhas.

\begin{tabular}{|c|c|c|c|c|c|c|c|c|c|}
\hline Fragmento & Área $\left(\mathbf{k m}^{2}\right)$ & $\begin{array}{c}\text { Perímetro } \\
(\mathbf{k m})\end{array}$ & IC & dIIC-50 & dIIC-100 & dIIC-500 & $\begin{array}{l}\text { dIIC- } \\
1000\end{array}$ & $\begin{array}{c}\text { dIIC- } \\
2000\end{array}$ & $\begin{array}{c}\text { dIIC } \\
\text { médio }\end{array}$ \\
\hline 1 & 27,140985 & 53,066 & 0,121 & 54,8977 & 54,8977 & 62,7793 & 60,0790 & 55,5054 & 57,6318 \\
\hline 2 & 5,418185 & 25,132 & 0,108 & 2,1878 & 2,1878 & 30,8062 & 29,4812 & 27,2369 & 18,3800 \\
\hline 3 & 17,577696 & 47,557 & 0,098 & 23,0265 & 23,0264 & 37,7314 & 36,1085 & 33,3597 & 30,6505 \\
\hline 4 & 7,880859 & 29,595 & 0,113 & 4,6286 & 4,6286 & 3,2445 & 6,3016 & 8,9940 & 5,5595 \\
\hline $5^{*}$ & 0,031709 & 0,886 & 0,507 & 0,0001 & 0,0001 & 0,0022 & 0,0161 & 0,0291 & 0,0095 \\
\hline $6^{*}$ & 0,005087 & 0,317 & 0,635 & 0,0000 & 0,0000 & 0,0002 & 0,0026 & 0,0047 & 0,0015 \\
\hline 7 & 1,145612 & 9,858 & 0,148 & 0,0978 & 0,0978 & 0,0707 & 0,8020 & 1,0753 & 0,4287 \\
\hline $8^{*}$ & 0,425036 & 2,65 & 0,760 & 0,0135 & 0,0135 & 0,0351 & 0,0336 & 0,3018 & 0,0795 \\
\hline 9 & 1,155779 & 6,966 & 0,299 & 0,0996 & 0,0996 & 0,0954 & 0,0913 & 1,3747 & 0,3521 \\
\hline 10 & 0,673049 & 4,88 & 0,355 & 0,0338 & 0,0338 & 0,0395 & 0,5050 & 0,7466 & 0,2717 \\
\hline 11 & 0,414106 & 2,857 & 0,637 & 0,0128 & 0,0128 & 0,0239 & 2,9254 & 0,4505 & 0,6851 \\
\hline $12^{*}$ & 0,026328 & 0,89 & 0,417 & 0,0001 & 0,0001 & 0,0013 & 0,0198 & 0,0252 & 0,0093 \\
\hline 13 & 8,05398 & 17,884 & 0,316 & 4,8342 & 4,8342 & 3,3887 & 6,0433 & 11,8826 & 6,1966 \\
\hline 14 & 2,936337 & 12,477 & 0,237 & 0,6426 & 0,6426 & 0,4884 & 0,9625 & 2,5105 & 1,0493 \\
\hline 15 & 0,247806 & 2,704 & 0,426 & 0,0046 & 0,0046 & 0,0412 & 0,0658 & 0,2629 & 0,0758 \\
\hline $16^{*}$ & 0,032735 & 0,703 & 0,831 & 0,0001 & 0,0001 & 0,0055 & 0,0086 & 0,0338 & 0,0096 \\
\hline 17 & 3,171021 & 13,699 & 0,212 & 0,7494 & 0,7494 & 0,5307 & 1,0030 & 3,4525 & 1,2970 \\
\hline $18^{*}$ & 0,659534 & 6,335 & 0,206 & 0,0324 & 0,0324 & 0,0227 & 0,0217 & 1,4107 & 0,3040 \\
\hline $19^{*}$ & 0,011756 & 0,643 & 0,357 & 0,0000 & 0,0000 & 0,0000 & 0,0007 & 0,8960 & 0,1793 \\
\hline $20^{*}$ & 0,023533 & 0,796 & 0,466 & 0,0001 & 0,0001 & 0,0000 & 0,0026 & 0,0131 & 0,0032 \\
\hline $21^{*}$ & 0,065239 & 1,024 & 0,781 & 0,0003 & 0,0003 & 0,0047 & 0,0054 & 0,0363 & 0,0094 \\
\hline $22^{*}$ & 1,321593 & 7,04 & 0,335 & 0,1302 & 0,1302 & 0,0957 & 0,1147 & 0,9605 & 0,2863 \\
\hline $23^{*}$ & 0,006956 & 0,315 & 0,877 & 0,0000 & 0,0000 & 0,0000 & 0,0210 & 0,0034 & 0,0049 \\
\hline $24^{*}$ & 0,018611 & 0,529 & 0,836 & 0,0001 & 0,0001 & 0,0001 & 0,0014 & 0,0091 & 0,0022 \\
\hline $25^{*}$ & 0,048308 & 0,867 & 0,807 & 0,0003 & 0,0003 & 0,0002 & 0,0036 & 0,0237 & 0,0056 \\
\hline $26^{*}$ & 0,494258 & 4,82 & 0,267 & 0,0182 & 0,0182 & 0,0128 & 0,0313 & 0,2248 & 0,0611 \\
\hline $27^{*}$ & 0,060892 & 1,013 & 0,745 & 0,0003 & 0,0003 & 0,0002 & 0,0002 & 0,0219 & 0,0046 \\
\hline $28^{*}$ & 0,019246 & 0,561 & 0,767 & 0,0000 & 0,0000 & 0,0000 & 0,0002 & 0,0002 & 0,0001 \\
\hline $29^{*}$ & 0,267235 & 2,777 & 0,435 & 0,0053 & 0,0053 & 0,0037 & 0,0036 & 0,0033 & 0,0042 \\
\hline $30^{*}$ & 0,224746 & 2,357 & 0,508 & 0,0038 & 0,0038 & 0,0026 & 0,0027 & 0,0025 & 0,0031 \\
\hline $31^{*}$ & 0,032643 & 0,679 & 0,890 & 0,0001 & 0,0001 & 0,0001 & 0,0001 & 0,0038 & 0,0008 \\
\hline $32^{*}$ & 0,002288 & 0,208 & 0,660 & 0,0000 & 0,0000 & 0,0000 & 0,0000 & 0,0003 & 0,0001 \\
\hline 33 & 0,21588 & 2,553 & 0,416 & 0,0035 & 0,0035 & 0,0024 & 0,0230 & 0,0359 & 0,0137 \\
\hline 34 & 1,426084 & 10,284 & 0,169 & 0,1516 & 0,1516 & 0,2169 & 0,2568 & 0,2673 & 0,2088 \\
\hline $35^{*}$ & 0,004234 & 0,244 & 0,890 & 0,0000 & 0,0000 & 0,0000 & 0,0213 & 0,0007 & 0,0044 \\
\hline 36 & 0,902957 & 5,467 & 0,380 & 0,0608 & 0,0608 & 0,0426 & 0,1719 & 0,1692 & 0,1010 \\
\hline 37 & 1,484792 & 10,396 & 0,173 & 0,1643 & 0,1643 & 0,2258 & 0,3405 & 0,2728 & 0,2336 \\
\hline $38^{*}$ & 0,028311 & 0,867 & 0,473 & 0,0001 & 0,0001 & 0,0096 & 0,0138 & 0,0127 & 0,0073 \\
\hline $39^{*}$ & 0,036934 & 1,137 & 0,359 & 0,0001 & 0,0002 & 0,0283 & 0,0180 & 0,0166 & 0,0126 \\
\hline 40 & 9,664724 & 29,211 & 0,142 & 6,9612 & 6,9612 & 4,9078 & 4,7013 & 4,3434 & 5,5749 \\
\hline 41 & 0,208765 & 2,027 & 0,638 & 0,0032 & 0,0032 & 0,0023 & 0,0022 & 0,0492 & 0,0120 \\
\hline 42 & 3,389933 & 10,322 & 0,400 & 0,8564 & 0,8564 & 0,9997 & 0,9567 & 0,9311 & 0,9201 \\
\hline 43 & 2,2553 & 13,652 & 0,152 & 0,3791 & 0,3791 & 0,6651 & 0,6365 & 0,6025 & 0,5325 \\
\hline Média & 2,307234 & 8,099 & 0,450 & 2,3256 & 2,3256 & 3,4076 & 3,5302 & 3,6641 & 3,0506 \\
\hline 44 & 6,830301 & 20,566 & 0,203 & 3,4768 & 3,4768 & 2,8544 & 5,7923 & 6,9696 & 4,5140 \\
\hline 45 & 10,499245 & 18,503 & 0,385 & 8,2152 & 8,2152 & 5,7587 & 5,5110 & 5,0914 & 6,5583 \\
\hline 46 & 8,91234 & 14,719 & 0,517 & 5,9195 & 5,9195 & 4,1494 & 3,9710 & 3,6687 & 4,7256 \\
\hline 47 & 4,599478 & 11,266 & 0,455 & 7,3059 & 7,3059 & 5,2377 & 6,7827 & 13,1032 & 7,9471 \\
\hline
\end{tabular}



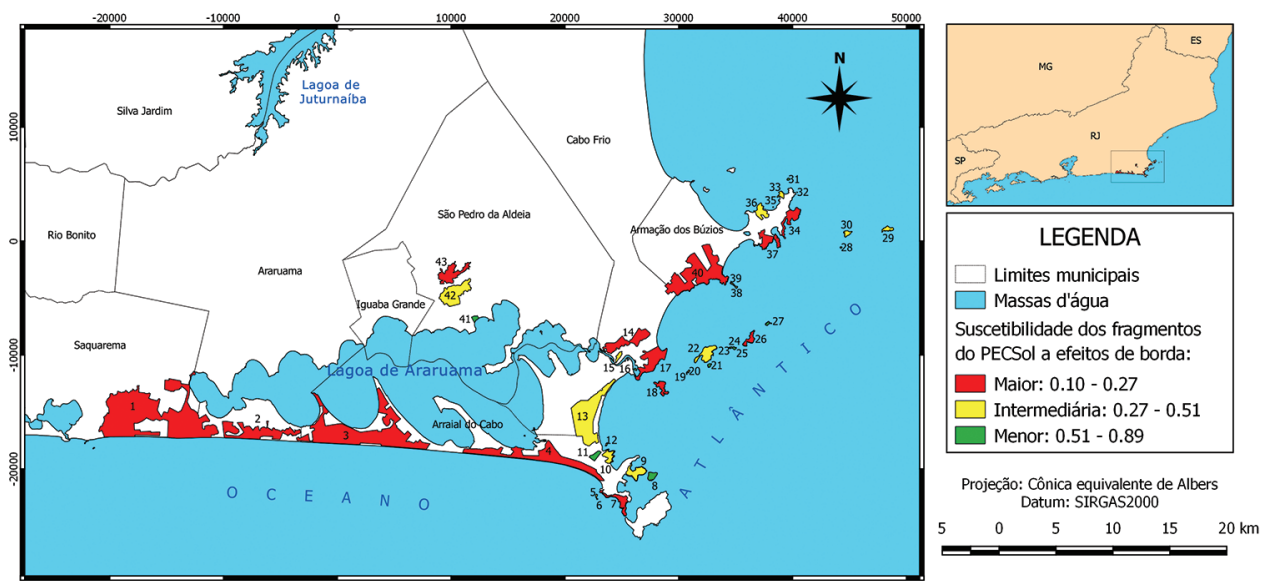

Figura 5. Suscetibilidade dos fragmentos do Parque Estadual da Costa do Sol (PECSol), Rio de Janeiro, Brasil, a efeitos de borda de acordo com seus Índices de Circularidade (IC).

e não-ilhas deve-se à necessidade, quando da demarcação das áreas de vegetação remanescente que comporiam o parque, de se desviar dos elementos antrópicos já consolidados na paisagem, como as pastagens, salinas e assentamentos urbanos, criando assim diversas reentrâncias nos desenhos dos polígonos. As ilhas, em contrapartida, são cercadas apenas por água e possuem naturalmente formas mais arredondadas, o que contribui para seus bons valores de IC.

Ressalta-se que apenas o formato de um fragmento não permite dizer de fato o quanto ele é acometido por efeitos de borda. Além do formato, também deve se considerar seu tamanho (Laurance e Yensen 1991). Fragmentos grandes com formatos irregulares podem ter uma área central livre de efeitos de borda extensa, assim como fragmentos circulares pequenos podem ser fortemente afetados por estes efeitos. Além disso, diferentes tipos de efeitos de borda possuem diferentes capacidades de penetração no interior de um fragmento (Laurance et al.2002). Assim, para identificar as porções dos fragmentos que realmente são acometidas por efeitos de borda em uma paisagem com grande heterogeneidade de habitats como o CDVCF, onde há formações vegetais desde restinga herbácea a áreas densas de floresta, um intenso esforço de campo seria necessário. Ainda assim, as métricas apontam áreas reduzidas nos fragmentos mais circulares e formatos muito irregulares nos fragmentos maiores, o que é indício de uma alta suscetibilidade a efeitos de borda no Parque Estadual da Costa de Sol como um todo.

\section{Conectividade e disponibilidade de habitat}

O valor médio das menores distâncias entre os fragmentos do PECSol é de 451,6m, o que significa que o parque possui uma boa conectividade para espécies com capacidade de dispersão igual ou maior que este valor. Aquelas com capacidade de dispersão menor, entretanto, tendem a ter suas populações isoladas em determinados fragmentos. Ressalta-se, porém, que estas distâncias foram calculadas de acordo com o menor caminho em 

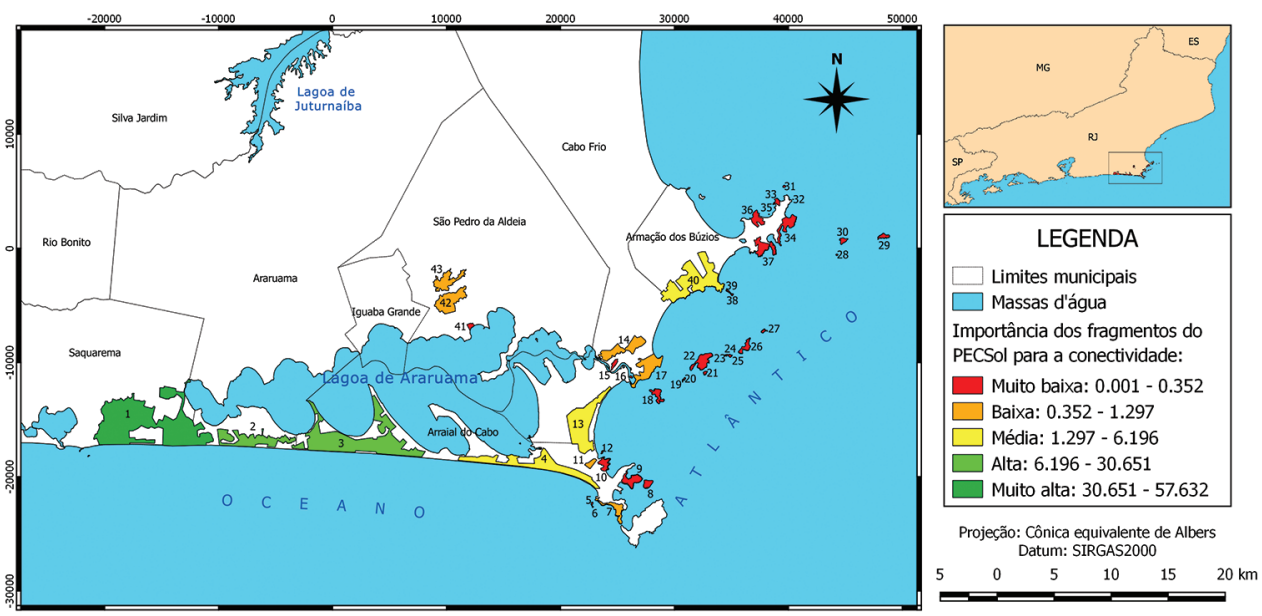

Figura 6. Importância dos fragmentos do Parque Estadual da Costa do Sol (PECSol) para a conectividade e disponibilidade de habitat na região do Centro de Diversidade Vegetal de Cabo Frio, Rio de Janeiro, Brasil.

linha reta entre os fragmentos. A distância, todavia, não é o único fator determinante no sucesso da dispersão de um organismo. A matriz entre as manchas de habitat também influencia esse sucesso, sendo ela mais ou menos permeável de acordo com seu tipo e com a espécie focal (Gustafson e Gardner 1996; Ewers e Didham 2006). Um aumento na heterogeneidade da matriz também leva a uma maior complexidade no movimento dos organismos entre fragmentos (Bender e Fahrig 2005). Conforme se constatou na análise da Zona de Amortecimento do PECSol (Figura 4), as áreas circundantes aos fragmentos podem ser ambientes muito inóspitos para a travessia de organismos, especialmente os não alados, o que pode fazer com que eles tenham que percorrer distâncias maiores de um fragmento para outro para esquivar-se dos elementos antrópicos da paisagem.

O valor médio do Índice Integral de Conectividade (IIC) para o PECSol, obtido a partir das análises com cinco valores de dispersão distintos $(50 \mathrm{~m}, 100 \mathrm{~m}, 500 \mathrm{~m}$, $1.000 \mathrm{~m}$ e $2.000 \mathrm{~m}$ ), foi de 0,00077894 . Este valor é consideravelmente baixo, indicando que o PECSol sozinho exerce uma função pouco eficaz na disponibilidade de habitat para as espécies que vivem no CDVCF. Os fragmentos que apresentaram os maiores valores médios de dIIC, em ordem decrescente, foram: 1, 3, 2, 13, 40 e 4. Os três primeiros, todos do Núcleo Massambaba, apresentaram valores entre $18 \%$ e $58 \%$, o que denota uma importância maior deste Núcleo em comparação aos demais no quesito de disponibilidade de habitat, conforme se observa na Figura 6. Os três fragmentos seguintes tiveram valores entre 5,5\% e 6,2\% e todos os demais apresentaram dIIC inferior a 1,3\% (ver Tabela 1). Esses dados podem ser úteis ao Poder Público para o planejamento e a gestão do PECSol, permitindo identificar quais fragmentos merecem maior esforço de conservação, sendo possível também simular cenários com a recuperação de áreas degradadas ou com o estabelecimento de corredores ecológicos (Pascual-Hortal e Saura 2006).

Deve-se destacar que esta análise de conectividade levou em conta apenas as áreas pertencentes ao PECSol e as considerou inteiras como áreas naturais, o que 
não necessariamente é verdadeiro. Para realizar uma análise diagnóstica do estado atual de conectividade do CDVCF seria necessário mapear todas as áreas naturais remanescentes, independente de estarem sob algum regime de proteção. O objetivo dessa análise, porém, foi de avaliar qual a contribuição da implementação do PECSol para a conectividade do CDVCF.

\section{Importância de outras áreas não inseridas no Parque Estadual da Costa do Sol}

Há quatro extensas áreas de vegetação natural no CDVCF que não estão sob regime de proteção por Unidade de Conservação de Proteção Integral: a Ilha de Cabo Frio, a porção vegetada da Base Aérea Naval de São Pedro da Aldeia (BAeNSPA), a área de restinga na Estação Radiogoniométrica da Marinha em Campos Novos (ERMCN), e as Dunas do Peró. Se incorporadas ao PECSol, o valor médio do IIC subiria para 0,00096636, ou seja, essas áreas promoveriam um aumento na sua conectividade e disponibilidade de habitat de $24 \%$. Seus valores de dIIC variam entre $4,5 \%$ e $8 \%$, superiores à maior parte dos fragmentos do PECSol (ver Tabela 1), o que revela a alta importância dessas áreas para a biodiversidade da região (Figura 7).

A Ilha de Cabo Frio, a BAeNSPA e a ERMCN são de responsabilidade da Marinha do Brasil, com rigidez na restrição ao acesso, o que contribui para o bom estado de conservação dessas áreas. Porém, o uso pela Marinha não garante a mesma perpetuidade de proteção que a conversão em Unidade de Conservação conferiria, visto que se as atividades da Marinha forem encerradas nessas áreas elas ficarão vulneráveis a usos mais intrusivos. A adesão destas áreas ao PECSol, entretanto, talvez gerasse conflitos de uso com as atividades da Marinha. Ainda assim, recomenda-se ao Poder Público estudar uma maneira de incluir essas áreas em Unidades de Conservação sem inviabilizar a presença da Marinha. A criação de uma nova categoria de Unidade de Conservação em áreas tuteladas às Forças Armadas, como sugerido por Guimarães e Braga (2012), pode ser uma boa alternativa para garantir uma proteção duradoura destas áreas.

As Dunas do Peró apresentaram o melhor dIIC dentre as quatro áreas analisadas (ver Tabela 1), sendo um importante elemento conector da paisagem. Elas estão inseridas nos limites da Área de Proteção Ambiental Estadual do Pau-Brasil, uma UC de Uso Sustentável. Porém, está prevista para esta área a construção de um enorme complexo turístico-residencial, inicialmente chamado de "Resort Peró", depois de "Reserva do Peró" e atualmente de "Costa do Peró". Esse empreendimento, entretanto, vem tendo sua legitimidade contestada há mais de 10 anos devido a diversas irregularidades em seu processo de licenciamento (Mello et al. 2015). Suas obras continuaram, à época desse estudo, suspensas pela Justiça Federal.

Destaca-se aqui, além dos dispositivos legais citados por Mello et al. (2015), a existência da lei estadual $n^{\circ} 1.807$, de 03 de abril de 1991, que determina:

Art. $1^{\circ}$ - Ficam criados os "Parques das Dunas" em todo o Estado do Rio de Janeiro.

Art. $2^{\circ}$ - Os parques a que se refere o artigo $1^{\circ}$ deverão ter como área as regiões onde existirem dunas. 

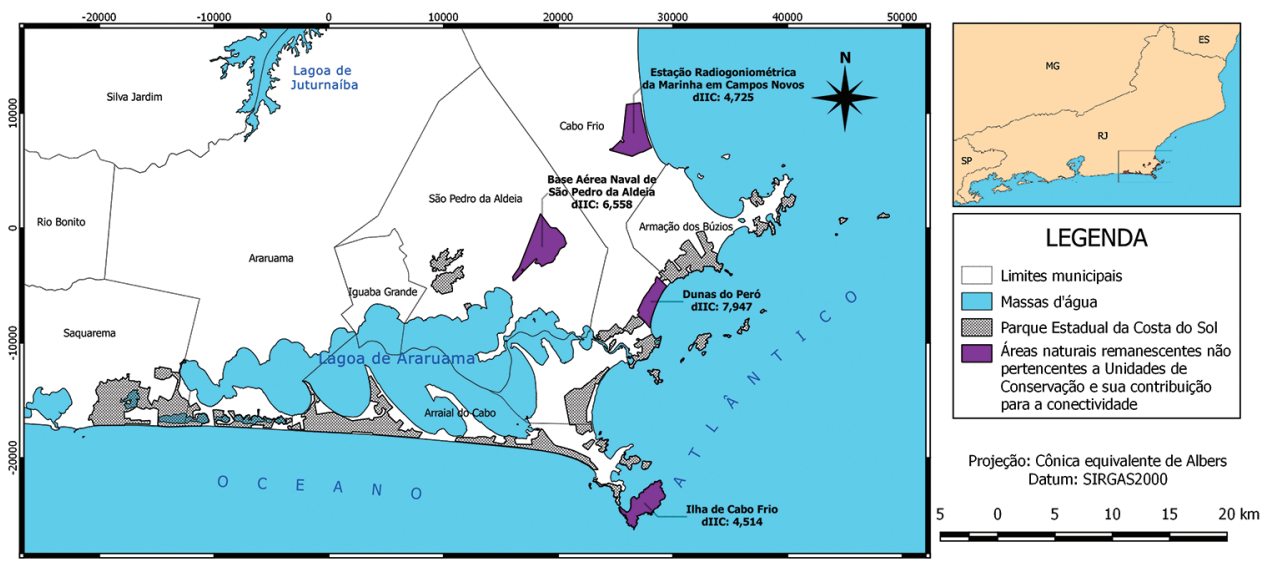

Figura 7. Localização das quatro áreas naturais analisadas em relação ao Parque Estadual da Costa do Sol (PECSol), e seus valores de importância para a conectividade e disponibilidade de habitat na região do Centro de Diversidade Vegetal de Cabo Frio, Rio de Janeiro, Brasil.

Art. $3^{\circ}$ - As regiões referidas no artigo $2^{\circ}$ deverão ser demarcadas “in loco", mapeadas em escala adequada e fiscalizadas pela Secretaria de Meio Ambiente.

Art. $4^{\circ}$ - Não se permitirá o desmembramento, construção de prédios ou expansão de construções existentes nas áreas referidas no artigo $2^{\circ}$. (Rio de Janeiro 1991)

O próprio decreto de criação do PECSol elenca como uma de suas justificativas que este deve considerar “a criação do Parque das Dunas do Estado do Rio de Janeiro, pela Lei Estadual n 1.807, de 03 de abril de 1991, que necessita de demarcação in loco, mapeamento e fiscalização do órgão ambiental estadual" (Rio de Janeiro 2011). Ainda assim, tanto as dunas do Peró como as de Tucuns não foram incluídas no seu desenho original. As dunas de Tucuns já se encontravam degradadas quando o PECSol foi instituído, devido à construção de um grande empreendimento turístico sobre elas (Corrêa e Fontenelle 2012). Quanto às Dunas do Peró, para que sua proteção seja garantida conforme previsto em lei, recomenda-se a revogação das licenças do empreendimento "Costa do Peró" e a sua inclusão nos limites do PECSol ou em outra UC de Proteção Integral.

\section{Considerações finais}

As análises das pressões das atividades no entorno dos fragmentos sobre eles exercidas, dos seus tamanhos e formatos e sua suscetibilidade a efeitos de borda, e da sua conectividade dentro da paisagem apontam que o Parque Estadual da Costa do Sol, por si só e da maneira como foi concebido, não demonstra ser um instrumento efetivo na proteção da biodiversidade única do Centro de Diversidade Vegetal de Cabo Frio. É pouco provável que todas as populações da fauna e da flora da região consigam se manter a longo prazo caso as áreas protegidas pelo Parque Estadual da Costa do Sol em sua configuração atual se tornem os últimos remanescentes de habitat natural da região. 
É importante destacar, porém, que a criação do Parque Estadual da Costa do Sol não foi o único esforço de conservação adotado para o Centro de Diversidade Vegetal de Cabo Frio. A fim de alcançar a proteção efetiva da biodiversidade da região é fundamental que haja uma gestão colaborativa de todos os órgãos e entidades responsáveis pelas demais Unidades de Conservação ali implementadas, sejam nacionais, estaduais ou municipais, de Uso Sustentável ou Proteção Integral. Para tal, recomenda-se a criação de um Mosaico de Unidades de Conservação da região, instrumento de gestão integrada previsto no Sistema Nacional de Unidades de Conservação (Brasil 2000). Também, a recuperação de áreas de Reserva Legal e de Preservação Permanente das propriedades rurais da região registradas no Cadastro Ambiental Rural (CAR), em acordo com a lei de proteção da vegetação nativa (Brasil 2012), deve ser planejada de maneira que contribua com o aumento da conectividade com as demais áreas naturais. Por fim, a criação de novas Unidades de Conservação ou a inclusão de mais áreas no Parque Estadual da Costa do Sol, como as quatro abordadas neste estudo, também é recomendada para promover uma maior proteção à biodiversidade local.

\section{Agradecimentos}

Agradecemos a Claudio Belmonte de Athayde Bohrer e Felipe Mendes Cronemberger pela disponibilização dos dados necessários a essa pesquisa, e a Cyl Farney Catarino de Sá, Julia Celia Mercedes Strauch e aos revisores anônimos pelos importantes comentários tecidos em versões anteriores deste manuscrito.

\section{Referências}

Ab’Saber AN (1977) Espaços ocupados pela expansão dos climas secos na América do Sul, por ocasião dos períodos glaciais quaternários. Paleoclimas 3: 1-18.

Araujo DSD (1997) Cabo Frio Region. In: Davis SD, Heywood VH, Herrera-Macbryde O, Villa-Lobos J, Hamilton AC (Eds) Centres of Plant Diversity: A Guide and Strategy for Their Conservation. Volume 3: The Americas. IUCN Publications Unit, Cambridge, 373-375.

Barbiére EB (1975) Ritmo climático e extração do sal em Cabo Frio. Revista Brasileira de Geografia 37(4): 23-109.

Barbiére EB (1997) Flutuações climáticas em Cabo Frio. Revista do Departamento de Geografia 11: 95-112. https://doi.org/10.7154/RDG.1997.0011.0008

Bender DJ, Fahrig L (2005) Matrix structure obscures the relationship between interpatch movement and patch size and isolation. Ecology 86(4): 1023-1033. https://doi. org/10.1890/03-0769

Bohrer CBA, Dantas HGR, Cronemberger FM, Vicens RS, Andrade SF (2009) Mapeamento da vegetação e uso do solo no Centro de Diversidade Vegetal de Cabo Frio. Rodriguésia 60(1): 1-24. https://doi.org/10.1590/2175-7860200960101 
Brasil (2000) Lei $n^{\circ} 9.985$ de 18 de julho de 2000. Regulamenta o art. 225, $₫ 1^{\circ}$, incisos I, II, III e VII da Constituição Federal, institui o Sistema Nacional de Unidades de Conservação da Natureza e dá outras providências. Diário Oficial da União 138(138): 1-6.

Brasil (2012) Lei n 12.651 de 25 de maio de 2012. Dispõe sobre a proteção da vegetação nativa; altera as Leis $\mathrm{n}^{\circ} \mathrm{s} 6.938$, de 31 de agosto de 1981, 9.393, de 19 de dezembro de 1996, e 11.428, de 22 de dezembro de 2006; revoga as Leis $\mathrm{n}^{\circ} \mathrm{s} 4.771$, de 15 de setembro de 1965, e 7.754, de 14 de abril de 1989, e a Medida Provisória $n^{\circ} 2.166-67$, de 24 de agosto de 2001; e dá outras providências. Diário Oficial da União 149(102): 1-8.

Coe HHG, Carvalho CN, Souza LOF, Soares A (2007) Peculiaridades ecológicas da região de Cabo Frio, RJ. Revista Tamoios 3(2).

Corrêa WB, Fontenelle TH (2012) O Parque Estadual da Costa do Sol: Contextualização e críticas ao processo de instituição. Revista Geonorte 3(4): 1150-1160.

Deetz CH, Adams OS (1921) Elements of Map Projection with Applications to Map and Chart Construction. U.S. Government Printing Office, Washington, 163 pp.

Ewers RM, Didham RK (2006) Confounding factors in the detection of species responses to habitat fragmentation. Biological Reviews of the Cambridge Philosophical Society 81(1): 117-142. https://doi.org/10.1017/S1464793105006949

García-Feced C, Saura S, Elena-Rosselló R (2011) Improving landscape connectivity in forest districts: A two-stage process for prioritizing agricultural patches for reforestation. Forest Ecology and Management 261(1): 154-161. https://doi.org/10.1016/j. foreco.2010.09.047

Guimarães HB, Braga RAP (2012) O Sistema Nacional de Unidades de Conservação e as matas tuteladas ao Exército Brasileiro: proposta de criação de uma nova categoria. Âmbito jurídico 96.

Gustafson EJ, Gardner RH (1996) The effect of landscape heterogeneity on the probability of patch colonization. Ecology 77(1): 94-107. https://doi.org/10.2307/2265659

Hargis CD, Bissonette JA, David JL (1998) The behavior of landscape metrics commonly used in the study of habitat fragmentation. Landscape Ecology 13(3): 167-186. https:// doi.org/10.1023/A:1007965018633

Jenks GF (1967) The data model concept in statistical mapping. International yearbook of cartography 7: 186-190.

Laurance WF, Yensen E (1991) Predicting the impacts of edge effects in fragmented habitats. Biological Conservation 55(1): 77-92. https://doi.org/10.1016/0006-3207(91)90006-U Laurance WF, Lovejoy TE, Vasconcelos HL, Bruna EM, Didham RK, Stouffer PC, Gascon C, Bierregaard RO, Laurance SG, Sampaio E (2002) Ecosystem decay of amazonian forest fragments: A 22-year investigation. Conservation Biology 16(3): 605-618. https://doi. org/10.1046/j.1523-1739.2002.01025.x

Lerner F, Jeronymo CAL (2017) Entre salinas, moradias e resort: Conflitos de uso e cobertura da terra na Área de Proteção Ambiental de Massambaba, Rio de Janeiro, Brasil. Caderno de Geografia 27(50): 534-556. https://doi.org/10.5752/p.2318-2962.2017v27n50p534

Li H, Wu J (2004) Use and misuse of landscape indices. Landscape Ecology 19(4): 389-399. https://doi.org/10.1023/B:LAND.0000030441.15628.d6 
Li W, Goodchild MF, Church R (2013) An efficient measure of compactness for two-dimensional shapes and its application in regionalization problems. International Journal of Geographical Information Science 27(6): 1227-1250. https://doi.org/10.1080/1365881 6.2012 .752093

Lovejoy TE, Bierregaard R, Rylands AB, Malcolm JR, Quintela CE, Harper LH, Brown Jr KS, Powell GVN, Schubart HOR, Hays MB (1986) Edge and other effects of isolation on Amazon forest fragments. In: Soulé ME (Ed.) Conservation biology: the science of scarcity and diversity. Sinauer, Sunderland, 257-285.

MacArthur RH, Wilson EO (1967) The theory of island biogeography. Princeton University, Princeton, $203 \mathrm{pp}$.

Mello DS, Martins MB, Neffa E (2015). Vaudeville ambiental: um estudo sobre estratégias e táticas em licenciamento de empreendimentos, o caso do Campo de Dunas do Peró, Cabo Frio-RJ. In: Monica EF, Rangel TLV, Rodrigues WO, Filho WM (Eds) $5^{\circ}$ Seminário Interdisciplinar em Sociologia e Direito n. 5 v. 5, Niterói (Brasil), outubro de 2015. Ed. PPGSD, Niterói, 42-61.

Metzger JP (2001) O que é ecologia de paisagens? Biota Neotropica 1(1-2): 1-9. https://doi. org/10.1590/S1676-06032001000100006

Osserman R (1978) Isoperimetric inequality. Bulletin of the American Mathematical Society 84(6): 1182-1238. https://doi.org/10.1090/S0002-9904-1978-14553-4

Pascual-Hortal L, Saura S (2006) Comparison and development of new graph-based landscape connectivity indices: Towards the priorization of habitat patches and corridors for conservation. Landscape Ecology 21(7): 959-967. https://doi.org/10.1007/s10980006-0013-Z

Pimm SL, Raven P (2000) Biodiversity: Extinction by numbers. Nature 403(6772): 843-845. https://doi.org/10.1038/35002708

Rio de Janeiro (1991) Lei n 1807, de 03 de abril de 1991. Dispõe sobre a criação dos "Parques das Dunas" em todo o Estado. Diário Oficial do Estado do Rio de Janeiro 12/04/91.

Rio de Janeiro (2011) Decreto $n^{\circ} 42.929$ de 18 de abril de 2011. Cria o Parque Estadual da Costa do Sol e dá outras providências. Diário Oficial do Estado do Rio de Janeiro 37(72): 1-5.

Saura S, Rubio L (2010) A common currency for the different ways in which patches and links can contribute to habitat availability and connectivity in the landscape. Ecography 33: 523-537. https://doi.org/10.1111/j.1600-0587.2009.05760.x

Taylor PD, Fahrig L, Henein K, Merriam G (1993) Connectivity is a vital element of landscape structure. Oikos 68(3): 571-573. https://doi.org/10.2307/3544927

Wittemyer G, Elsen P, Bean WT, Burton ACO, Brashares JS (2008) Accelerated human population growth at protected area edges. Science 321(5885): 123-126. https://doi. org/10.1126/science.1158900

Zimmermann TG (2016) Potencial de Invasão das Restingas por Casuarina equisetifolia L.: Fatores que Limitam a Regeneração da Vegetação. Tese de doutorado, Rio de Janeiro, Brasil: Escola Nacional de Botânica Tropical. 